\title{
Exchange Coupling of Paramagnetic Ions in Polyoxometalate Matrix. Density Functional Study of Diiron(III) Substituted $\gamma$-Silicotungstate
}

\author{
Ekaterina M. Zueva, Henry Chermette, Serguei A. Borshch
}

\section{Supporting Information}

Table 1. Optimized Cartesian coordinates (in $\AA$ ) for oxo-bridged polyanion I (the structure with the bond angle $\mathrm{Fe}-\mathrm{Fe}-\mathrm{OH}_{2}$ fixed at $95^{\circ}$ )

$\begin{array}{lrrr}\mathrm{Si} & .000000 & .000000 & .000000 \\ \mathrm{O} & -1.402736 & .000000 & -1.010527 \\ \mathrm{O} & 1.402736 & .000000 & -1.010527 \\ \mathrm{~W} & -3.609233 & .000000 & -.254135 \\ \mathrm{~W} & -1.912919 & -1.750035 & -2.614317 \\ \mathrm{~W} & -1.912919 & 1.750035 & -2.614317 \\ \mathrm{O} & -2.927853 & 1.474336 & .778990 \\ \mathrm{O} & -3.549237 & -1.266273 & -1.746181 \\ \mathrm{O} & -2.927853 & -1.474336 & .778990 \\ \mathrm{O} & -3.549237 & 1.266273 & -1.746181 \\ \mathrm{O} & -5.309688 & .000000 & .189674 \\ \mathrm{O} & -1.962185 & .000000 & -3.476932 \\ \mathrm{O} & .000000 & -1.803863 & -2.887549 \\ \mathrm{O} & -1.661341 & -2.871200 & -1.065981 \\ \mathrm{O} & -2.438410 & -2.873455 & -3.890448 \\ \mathrm{O} & .000000 & 1.803863 & -2.887549 \\ \mathrm{O} & -1.661341 & 2.871200 & -1.065981 \\ \mathrm{O} & -2.438410 & 2.873455 & -3.890448 \\ \mathrm{~W} & 3.609233 & .000000 & -.254135 \\ \mathrm{~W} & 1.912919 & 1.750035 & -2.614317 \\ \mathrm{~W} & 1.912919 & -1.750035 & -2.614317 \\ \mathrm{O} & 2.927853 & -1.474336 & .778990 \\ \mathrm{O} & 3.549237 & 1.266273 & -1.746181 \\ \mathrm{O} & 2.927853 & 1.474336 & .778990 \\ \mathrm{O} & 3.549237 & -1.266273 & -1.746181 \\ \mathrm{O} & 5.309688 & .000000 & .189674 \\ \mathrm{O} & 1.962185 & .000000 & -3.476932 \\ \mathrm{O} & 1.661341 & 2.871200 & -1.065981 \\ \mathrm{O} & 2.438410 & 2.873455 & -3.890448 \\ \mathrm{O} & 1.661341 & -2.871200 & -1.065981 \\ \mathrm{O} & 2.438410 & -2.873455 & -3.890448 \\ \mathrm{O} & .000000 & -1.460318 & .902903 \\ \mathrm{O} & .000000 & 1.460318 & .902903 \\ \mathrm{Fe} & .000000 & -1.344033 & 3.059640 \\ \mathrm{~W} & 1.721736 & -3.101968 & .913936 \\ \mathrm{~W} & -1.721736 & -3.101968 & .913936 \\ \mathrm{O} & 1.333770 & .000000 & 3.369792 \\ \mathrm{O} & 1.443038 & -2.781161 & 2.709723 \\ \mathrm{O} & -1.443038 & -2.781161 & 2.709723 \\ \mathrm{O} & .000000 & -1.548503 & 5.396742\end{array}$


O $.000000 \quad-4.016370$

.794946 
Table 1 (continued). Optimized Cartesian coordinates (in $\AA$ ) for oxo-bridged polyanion $\mathbf{I}$ (the structure with the bond angle $\mathrm{Fe}-\mathrm{Fe}-\mathrm{OH}_{2}$ fixed at $95^{\circ}$ )

$\begin{array}{lrrr}\mathrm{O} & 2.883846 & -4.443663 & 1.055366 \\ \mathrm{O} & -2.883846 & -4.443663 & 1.055366 \\ \mathrm{Fe} & .000000 & 1.344033 & 3.059640 \\ \mathrm{~W} & -1.721736 & 3.101968 & .913936 \\ \mathrm{~W} & 1.721736 & 3.101968 & .913936 \\ \mathrm{O} & -1.333770 & .000000 & 3.369792 \\ \mathrm{O} & -1.443038 & 2.781161 & 2.709723 \\ \mathrm{O} & 1.443038 & 2.781161 & 2.709723 \\ \mathrm{O} & .000000 & 1.548503 & 5.396742 \\ \mathrm{O} & .000000 & 4.016370 & .794946 \\ \mathrm{O} & -2.883846 & 4.443663 & 1.055366 \\ \mathrm{O} & 2.883846 & 4.443663 & 1.055366 \\ \mathrm{H} & -.722096 & -2.103945 & 5.666114 \\ \mathrm{H} & .722096 & -2.103945 & 5.666114 \\ \mathrm{H} & -.722096 & 2.103945 & 5.666114 \\ \mathrm{H} & .722096 & 2.103945 & 5.666114\end{array}$


Table 2. Optimized Cartesian coordinates (in $\AA$ ) for hydroxo-bridged polyanion II (the structure with the bond angle $\mathrm{Fe}-\mathrm{Fe}-\mathrm{OH}_{2}$ fixed at $95^{\circ}$ )

\begin{tabular}{|c|c|c|c|}
\hline Si & .000000 & .000000 & .000000 \\
\hline 0 & -1.366733 & .000000 & -1.023552 \\
\hline O & 1.366733 & .000000 & -1.023552 \\
\hline W & -3.606275 & .000000 & -.148790 \\
\hline W & -1.887772 & -1.709711 & -2.573814 \\
\hline W & -1.887772 & 1.709711 & -2.573814 \\
\hline O & -2.900520 & 1.450956 & .851580 \\
\hline O & -3.539132 & -1.267917 & -1.663776 \\
\hline O & -2.900520 & -1.450956 & .851580 \\
\hline O & -3.539132 & 1.267917 & -1.663776 \\
\hline O & -5.307061 & .000000 & .257628 \\
\hline O & -2.056495 & .000000 & -3.470610 \\
\hline 0 & .000000 & -1.702292 & -2.883628 \\
\hline O & -1.551334 & -2.816756 & -.994556 \\
\hline 0 & -2.539027 & -2.866447 & -3.744353 \\
\hline O & .000000 & 1.702292 & -2.883628 \\
\hline O & -1.551334 & 2.816756 & -. .994556 \\
\hline O & -2.539027 & 2.866447 & -3.744353 \\
\hline W & 3.606275 & .000000 & -. 148790 \\
\hline W & 1.887772 & 1.709711 & -2.573814 \\
\hline W & 1.887772 & -1.709711 & -2.573814 \\
\hline O & 2.900520 & -1.450956 & .851580 \\
\hline O & 3.539132 & 1.267917 & -1.663776 \\
\hline O & 2.900520 & 1.450956 & .851580 \\
\hline 0 & 3.539132 & -1.267917 & -1.663776 \\
\hline 0 & 5.307061 & .000000 & .257628 \\
\hline O & 2.056495 & .000000 & -3.470610 \\
\hline 0 & 1.551334 & 2.816756 & -.994556 \\
\hline O & 2.539027 & 2.866447 & -3.744353 \\
\hline O & 1.551334 & -2.816756 & -.994556 \\
\hline O & 2.539027 & -2.866447 & -3.744353 \\
\hline O & .000000 & -1.406496 & .968612 \\
\hline 0 & .000000 & 1.406496 & .968612 \\
\hline $\mathrm{Fe}$ & .000000 & -1.510000 & 3.111995 \\
\hline W & 1.726105 & -3.081217 & .908431 \\
\hline W & -1.726105 & -3.081217 & .908431 \\
\hline O & 1.306917 & -.000000 & 3.407326 \\
\hline O & 1.451996 & -2.707785 & 2.731528 \\
\hline O & -1.451996 & -2.707785 & 2.731528 \\
\hline O & .000000 & -1.704737 & 5.337851 \\
\hline O & .000000 & -3.984695 & .837821 \\
\hline O & 2.846785 & -4.457207 & 1.076746 \\
\hline O & -2.846785 & -4.457207 & 1.076746 \\
\hline $\mathrm{Fe}$ & .000000 & 1.510000 & 3.111995 \\
\hline W & -1.726105 & 3.081217 & .908431 \\
\hline W & 1.726105 & 3.081217 & .908431 \\
\hline O & -1.306917 & .000000 & 3.407326 \\
\hline
\end{tabular}


0

$-1.451996$

2.707785

2.731528 
Table 2 (continued). Optimized Cartesian coordinates (in $\AA$ ) for hydroxo-bridged polyanion II (the structure with the bond angle $\mathrm{Fe}-\mathrm{Fe}-\mathrm{OH}_{2}$ fixed at $95^{\circ}$ )

$\begin{array}{rrrr}\mathrm{O} & 1.451996 & 2.707785 & 2.731528 \\ \mathrm{O} & .000000 & 1.704737 & 5.337851 \\ \mathrm{O} & .000000 & 3.984695 & .837821 \\ \mathrm{O} & -2.846785 & 4.457207 & 1.076746 \\ \mathrm{O} & 2.846785 & 4.457207 & 1.076746 \\ \mathrm{H} & -.722096 & -2.260179 & 5.607223 \\ \mathrm{H} & .722096 & -2.260179 & 5.607223 \\ \mathrm{H} & -.722096 & 2.260179 & 5.607223 \\ \mathrm{H} & .722096 & 2.260179 & 5.607223 \\ \mathrm{H} & 2.142043 & .000000 & 2.857269 \\ \mathrm{H} & -2.142043 & .000000 & 2.857269\end{array}$


Table 3. Optimized Cartesian coordinates (in $\AA$ ) for oxo-bridged dimer III

$\begin{array}{lrrr}\mathrm{Fe} & .000000 & -1.386101 & .000000 \\ \mathrm{O} & 1.330855 & .000000 & .000000 \\ \mathrm{O} & -1.330855 & .000000 & .000000 \\ \mathrm{Fe} & .000000 & 1.386101 & .000000 \\ \mathrm{O} & .000000 & -1.996817 & -2.028820 \\ \mathrm{O} & .000000 & -1.996817 & 2.028820 \\ \mathrm{O} & .000000 & 1.996817 & 2.028820 \\ \mathrm{O} & .000000 & 1.996817 & -2.028820 \\ \mathrm{O} & 1.855302 & -2.661253 & .000000 \\ \mathrm{O} & -1.855302 & -2.661253 & .000000 \\ \mathrm{O} & 1.855302 & 2.661253 & .000000 \\ \mathrm{O} & -1.855302 & 2.661253 & .000000 \\ \mathrm{H} & -.822817 & -2.143945 & -2.562678 \\ \mathrm{H} & .822817 & -2.143945 & -2.562678 \\ \mathrm{H} & .822817 & -2.143945 & 2.562678 \\ \mathrm{H} & -.822817 & -2.143945 & 2.562678 \\ \mathrm{H} & -.822817 & 2.143945 & 2.562678 \\ \mathrm{H} & .822817 & 2.143945 & 2.562678 \\ \mathrm{H} & 2.091949 & -3.626913 & .000000 \\ \mathrm{H} & 2.662847 & -2.080938 & .000000 \\ \mathrm{H} & -2.091949 & -3.626913 & .000000 \\ \mathrm{H} & -2.662847 & -2.080938 & .000000 \\ \mathrm{H} & 2.091949 & 3.626913 & .000000 \\ \mathrm{H} & 2.662847 & 2.080938 & .000000 \\ \mathrm{H} & -2.091949 & 3.626913 & .000000 \\ \mathrm{H} & -2.662847 & 2.080938 & .000000 \\ \mathrm{H} & .822817 & 2.143945 & -2.562678 \\ \mathrm{H} & -.822817 & 2.143945 & -2.562678\end{array}$


Table 4. Optimized Cartesian coordinates (in $\AA$ ) for hydroxo-bridged dimer IV

$\begin{array}{lrrr}\mathrm{Fe} & .000000 & -1.643724 & .000000 \\ \mathrm{O} & 1.201388 & .000000 & .000000 \\ \mathrm{O} & -1.201388 & .000000 & .000000 \\ \mathrm{Fe} & .000000 & 1.643724 & .000000 \\ \mathrm{O} & .000000 & -2.071852 & -2.031580 \\ \mathrm{O} & .000000 & -2.071852 & 2.031580 \\ \mathrm{O} & .000000 & 2.071852 & 2.031580 \\ \mathrm{O} & .000000 & 2.071852 & -2.031580 \\ \mathrm{O} & 1.703233 & -2.966119 & .000000 \\ \mathrm{O} & -1.703233 & -2.966119 & .000000 \\ \mathrm{O} & 1.703233 & 2.966119 & .000000 \\ \mathrm{O} & -1.703233 & 2.966119 & .000000 \\ \mathrm{H} & -.818756 & -2.248900 & -2.580061 \\ \mathrm{H} & .818756 & -2.248900 & -2.580061 \\ \mathrm{H} & .818756 & -2.248900 & 2.580061 \\ \mathrm{H} & -.818756 & -2.248900 & 2.580061 \\ \mathrm{H} & -.818756 & 2.248900 & 2.580061 \\ \mathrm{H} & .818756 & 2.248900 & 2.580061 \\ \mathrm{H} & 1.601791 & -3.964980 & .000000 \\ \mathrm{H} & 2.682983 & -2.760546 & .000000 \\ \mathrm{H} & -1.601791 & -3.964980 & .000000 \\ \mathrm{H} & -2.682983 & -2.760546 & .000000 \\ \mathrm{H} & 1.601791 & 3.964980 & .000000 \\ \mathrm{H} & 2.682983 & 2.760546 & .000000 \\ \mathrm{H} & -1.601791 & 3.964980 & .000000 \\ \mathrm{H} & -2.682983 & 2.760546 & .000000 \\ \mathrm{H} & .818756 & 2.248900 & -2.580061 \\ \mathrm{H} & -.818756 & 2.248900 & -2.580061 \\ \mathrm{H} & 2.199363 & .000000 & .000000 \\ \mathrm{H} & -2.199363 & .000000 & .000000\end{array}$


Table 5. BS-state atomic spin densities for oxo- and hydroxo-bridged dimers III and IV, and polyanions I and II

\begin{tabular}{|c|c|c|c|c|c|c|c|c|c|}
\hline \multirow[b]{2}{*}{ atom } & \multicolumn{2}{|c|}{ III } & \multicolumn{2}{|c|}{ IV } & \multirow[b]{2}{*}{ atom } & \multicolumn{2}{|c|}{ I } & \multicolumn{2}{|c|}{ II } \\
\hline & left & right & left & right & & left & right & left & right \\
\hline $\mathrm{Fe}$ & 4.00 & -4.00 & 4.19 & -4.19 & $\mathrm{Fe}$ & 3.73 & -3.73 & 4.02 & -4.02 \\
\hline $\mathrm{O}_{\mathrm{eq}}$ & 0.02 & -0.02 & 0.08 & -0.08 & $\mathrm{O}_{\mathrm{eq}}$ & 0.04 & -0.04 & 0.18 & -0.18 \\
\hline $\mathrm{O}_{\mathrm{ax}}$ & 0.04 & -0.04 & 0.11 & -0.11 & $\mathrm{O}_{\mathrm{Si}}$ & 0.08 & -0.08 & 0.11 & -0.11 \\
\hline & & & & & $\mathrm{OH}_{2}$ & 0.01 & -0.01 & 0.02 & -0.02 \\
\hline
\end{tabular}




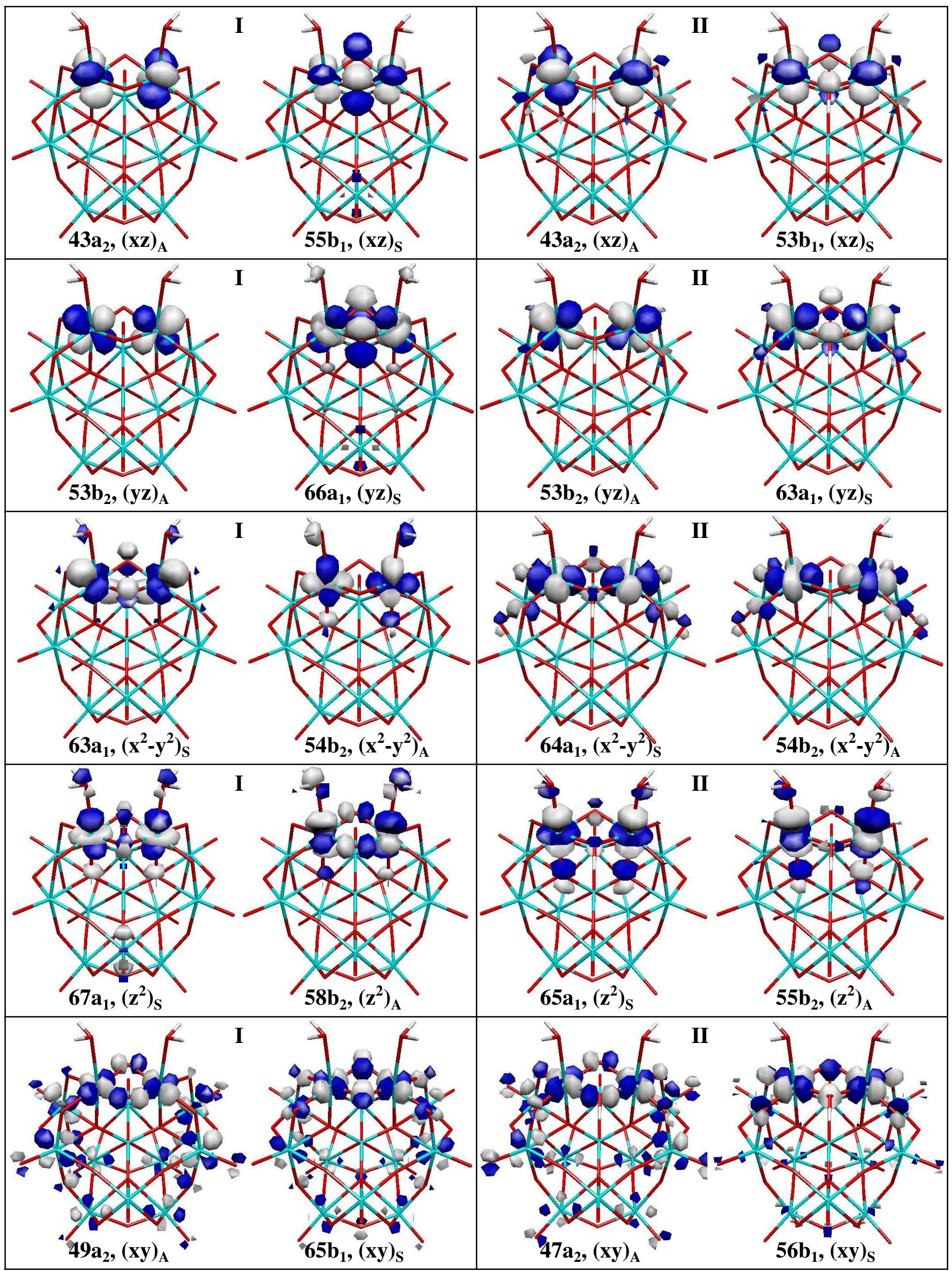


Figure 1. HS-state $\beta$-orbitals for oxo- and hydroxo-bridged polyanions I and II 